\title{
Age-related sensory deficits and their consequences
}

\author{
Jonathan E. Peelle
}

Department of Otolaryngology, Washington University in St. Louis, St. Louis MO USA

To appear as:

Peelle JE (In press) Age-related sensory deficits and their consequences. In: The Handbook of Cognitive Aging: A Life Course Perspective (Thomas and Gutchess, eds). Cambridge University Press.

Please address correspondence to:

Dr. Jonathan Peelle

Department of Otolaryngology

Washington University in Saint Louis

660 South Euclid, Box 8115

Saint Louis, MO 63110

email: ipeelle@wustl.edu 


\begin{abstract}
Changes in sensory systems are common as we get older, and become more likely with increasing age. In the auditory system, age-related changes are seen in domains such as auditory sensitivity, temporal processing, and spatial localization, which have significant effects on speech understanding. In vision, age related changes are seen in contrast sensitivity, scotopic processing, and visual processing speed, which have consequences for activities such as reading and driving. In addition to hearing and vision, aging is associated with changes in smell, taste, and balance. Beyond simple perceptual processing, age-related sensory changes can increase cognitive demands, requiring greater involvement of domain-general cognitive processes during perception that reduce resources available for other operations. Capturing individual variability in sensory changes and their consequences is an important part of understanding normal and pathological aging.
\end{abstract}

Key words: vision, driving, reading, hearing, speech, language, communication, sensory 


\subsection{Introduction}

Normal aging is frequently accompanied by changes in sensory ability (for reviews, see Gates and Mills, 2005; Owsley, 2016; Doty, 2018) that can directly interfere with perception, locomotion, and social interaction. However, age-related sensory declines can also have significant effects on cognitive processing that have broad implications for language, attention, and memory. In this chapter I review age-related changes in sensory systems and some of the most salient consequences of these changes. As in other aspects of development, an important theme in age-related sensory decline is the concept of individual differences. Older adults vary in their sensory processing, and this variability has been helpful in disentangling effects of aging from effects of sensory decline.

\subsection{Hearing}

\subsubsection{Age-Related Changes in Hearing}

The human auditory system is illustrated in Figure 4.1. Although hearing impairment has many etiologies, age-related hearing loss is common and affects $80 \%$ of adults over the age of 70 years (Cruickshanks et al., 1998). Age-related changes in hearing ability occur at all levels of the auditory system, from peripheral changes in the cochlea through to altered tuning in auditory cortex (Peelle and Wingfield, 2016). These changes manifest in several areas of auditory processing. 


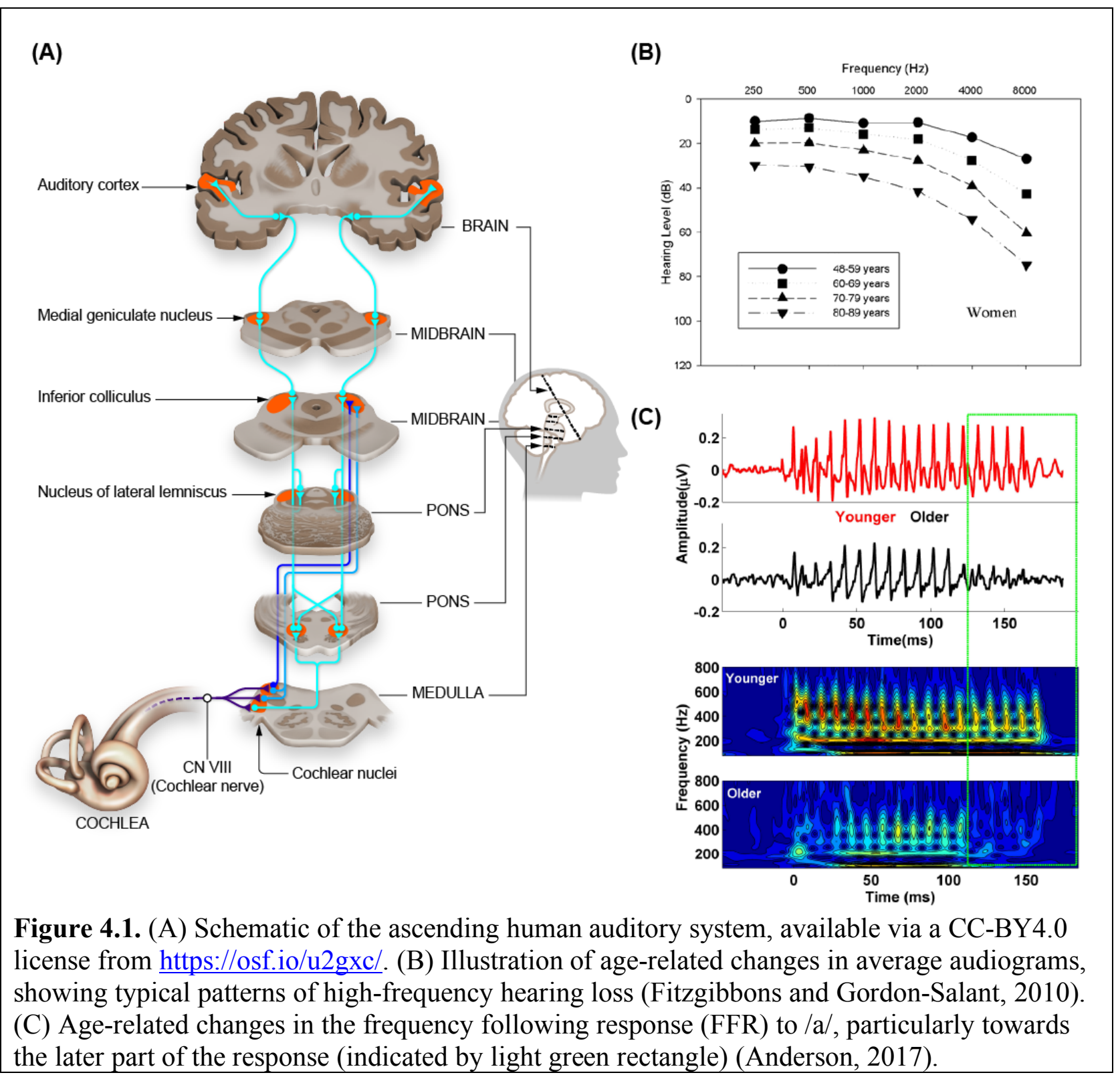

\subsubsection{Auditory Sensitivity}

Perhaps the most obvious age-related hearing changes occur due to a decrease in the number of outer hair cells, which lie along the basilar membrane and help translate acoustic energy to nerve signals. In aging, outer hair cell loss is preferentially seen in the region of the basilar membrane responsible for encoding high frequency information (Merchant and Nadol, 2010). Auditory sensitivity is typically assessed using pure-tone thresholds, in which tones of different frequencies are presented and participants are asked to indicate whether a tone was perceived or not. The pattern of high-frequency hearing loss commonly seen in older adulthood is illustrated in Figure 4.1B. Although this diminished sensitivity is strongly associated with a listener's ability to understand speech (Humes, 1996; Humes and Dubno, 2010), it does not capture all of the abilities required for understanding spoken language. One potential reason for this is the 
presence of more subtle synaptic dysfunction (Sergeyenko et al., 2013; Kujawa and Liberman, 2015), which may be invisible on standard threshold tests but can exert subtle effects on speech comprehension. However, other contributions are found in auditory processing beyond simple sound detection.

\subsubsection{Temporal Processing}

Aging is associated with difficulty with auditory temporal processing. One common approach to assessing temporal processing sensitivity is to use a gap detection task in which listeners are presented with a stimulus (such as a tone) and asked to indicate whether there were one or two sounds (or alternatively, listeners are presented with two items and asked to indicate which item contained the gap). When the distance between the two sounds (i.e., the "gap") is long, listeners can easily identify the presence of two sounds. At short gaps, however, older adults are less likely to perceive the gap (Moore et al., 1992). Schneider and colleagues (1994) presented young and older adult listeners with tones in a two-interval forced-choice procedure, and asked participants to indicate whether the first or second interval contained a gap. The average gap detection thresholds for the older adults were nearly twice as long as those of the young adults (6.3 $\mathrm{ms}$ for the older adults vs. $3.7 \mathrm{~ms}$ for the young adults) (see also Snell, 1997; Ozmeral et al., 2016). Because of the importance of precise timing information for speech perception, these changes in gap detection are presumed to carry over into difficulties with speech understanding.

A second way to measure temporal processing is to ask listeners to detect low-frequency (e.g., $2 \mathrm{~Hz}$ ) modulations of tonal carriers (e.g., 500-1500 Hz). Low frequency information may relate to speech features such as prosody and segmental cues (Rosen, 1992). One way to assess auditory processing is through dichotic listening tasks, in which different information is presented to the left and right ears. Listeners with hearing impairment perform more poorly on low frequency discrimination tasks (Buss et al., 2004), with larger age differences appearing for dichotic listening compared to monaural presentation (Grose and Mamo, 2012).

A third way in which temporal encoding accuracy has been assessed is through the measurement of auditory brain stem responses (ABRs) with EEG (Skoe and Kraus, 2010). ABRs measure electrical processing along the auditory pathway as recorded by surface electrodes; peaks reflect the summed activity of neurons as a particular time following a stimulus. Because ABRs are relatively small signals, data are typically averaged over a large number of trials. Thus, a reduction of a peak in an ABR response can indicate characteristics such as a lower rate of neural activity, or less consistency in the time-locking of neural activity to an acoustic stimulus, both of which are likely to have consequences for speech processing.

Although when they were first introduced ABR responses were recorded primarily to acoustically-simple stimuli (such as clicks), the technique has developed to be used for more complex stimuli (such as syllables). ABRs are closely related to the acoustic waveforms of presented stimuli, suggesting ABRs may be a noninvasive way of objectively assessing temporal coding precision in the auditory system. For example, the frequency following response is a time-locked neural response that peaks at the same frequency as the fundamental frequency (pitch) of the speech stimulus (Worden and Marsh, 1968). Various components of the ABR have been shown to be affected by age and hearing loss, showing representations of time-locked acoustic information in subcortical regions that are less accurate than those of young listeners with good hearing (Anderson et al., 2012; Anderson et al., 2013). An example of age-related change in the frequency-following response is shown in Figure 4.1C.

Consistent across these multiple types of assessment, aging is associated with poorer temporal auditory processing. Age-related differences in temporal processing have been 
associated with poorer speech perception (Pichora-Fuller and Souza, 2003; Gordon-Salant et al., 2006; Walton, 2010) and likely contribute to the challenges of speech comprehension in older adulthood.

\subsubsection{Sound Source Localization}

Knowing where a sound is coming from is critical for understanding speech in everyday situations. For example, when having a discussion at a noisy restaurant, it is useful to be able to disentangle speech produced by a conversational partner seated across the table from a discussion coming from another nearby table. For listeners with binaural hearing (that is, hearing from both ears), sound source localization is accomplished using a number of cues (Middlebrooks and Green, 1991). One important cue comes from interaural time differences (ITDs), which occur because sound from the environment will reach one ear before the other (unless the sound is equally distant from the two ears); the difference in timing provides information about where the sound is coming from (Carr and Konishi, 1990). Similarly, interaural level differences (ILDs) occur because a sound coming from off the midline will have different intensities at the two ears, again providing information as to its location.

Whereas ITDs and ILDs are the dominant cues for horizontal sound localization, highfrequency monaural cues contribute to both vertical sound localization and resolve front-back errors (Middlebrooks, 1992; Carlile et al., 1999). Experimentally, sound localization ability is typically tested with free-field presentation using multispeaker arrays positioned around a listener. Sounds are played from a speaker in the array and listeners indicate the location from which they heard the sound.

Abel and colleagues (2000) presented narrowband and broadband noises to young and older adults using a forced choice task, and found that older adults were less accurate at sound localization, with some indication of better performance on the left side of space. Comparable results were reported by Dobreva and colleagues (2011), who had participants indicate perceived location using a laser pointer. Again, older adults' errors of sound localization were larger than young adults in both the horizontal and vertical planes. For narrowband noise, the age-related effects were more apparent at $1250-1575 \mathrm{~Hz}$ than $250-500 \mathrm{~Hz}$, suggesting frequency-specific effects (for example, greater sensitivity at some frequencies that impacts localization ability).

\subsubsection{Consequences of Age-Related Changes in Hearing}

\subsubsection{Intelligibility of Speech in Noise}

Listeners frequently need to understand speech in the presence of background noise or competing talkers, and this is precisely the area in which age-related hearing loss has its most obvious effects. In laboratory settings, speech in noise comprehension is typically tested by presenting stimuli over headphones at various signal-to-noise ratios (SNRs). Compared to young adults, older adults have more difficulty understanding speech in noise, reflected in both intelligibility and memory scores (Pichora-Fuller et al., 1995; Humes, 1996; Tun and Wingfield, 1999). Although age-related changes in hearing can explain a great deal of older adults' difficulty understanding speech in noise, cognitive factors also play an important role (Humes and Dubno, 2010).

\subsubsection{Spatial Release from Masking}

Spatial release from masking refers to the benefit listeners typically experience when target speech and masking speech (such as a competing talker, or background noise) are separated in 
space. This benefit declines with both age and hearing loss. Glyde and colleagues (2013) investigated the contributions of ILDs and ITDs to spatial release from masking by altering standard head-related transfer functions (that is, mathematical estimates of how a head would affect acoustic signal to the left and right ear) to eliminate specific acoustic cues - in other words, selectively removing ILD or ITD information. Although the presence of ITDs or ILDs conferred some benefit, ITDs alone were less beneficial when ILD information was reduced. Given age-related changes in spatial localization associated with ILD and ITD information, we might expect older adults to experience less benefit from spatially-separated sources than do young adults. In keeping with this prediction, Gelfrand and colleagues (1988) found that agerelated hearing loss was the main factor affecting the amount of improvement achieved from spatial separation.

Gallun and colleagues (2013) used stimuli drawn from a Coordinate Response Measure (CRM) paradigm of the form: "Ready [CALL SIGN] go to [COLOR] [NUMBER] now" (Bolia et al., 2000) that were presented from speakers varying in their spatial separation. In the CRM task the directions are incidental to the task; the dependent measure is the number of words correct. The CRM provides a closed set of responses which not only aids scoring, but also provides enough unique combinations to allow presenting a large number of distinct sentences. The authors found that both individual differences in age and hearing loss were associated with identification accuracy in an adaptive task. Thus, age-related changes in hearing affect speech intelligibility in numerous ways, including through reducing the benefit most listeners experience when a target is separated from a competing acoustic stream.

\subsubsection{Cognitive Demands During Speech Understanding}

Although it is intuitive that reduced auditory processing abilities would make understanding speech more difficult, recent years have seen an increased interest in a less obvious consequence of hearing loss: the additional cognitive effort required to understand speech that is acoustically degraded. The general proposition is that if there is sufficient perceptual information, speech perception involves relatively little cognitive demand. However, if the acoustic signal is degraded - due to background noise, or age-related hearing loss - additional cognitive resources are needed to understand what has been said (Pichora-Fuller et al., 2016; Peelle, 2018). Given our limited capacity for cognitive processing, the additional requirements during perception thus take up resources that might have otherwise been used for other operations (such as remembering what we have heard) (Wingfield et al., 2005). A schematic of this framework is shown in Figure 4.2. The demand of a given situation depends both on the nature of the speech signal and the abilities of a given listener; the effort allocated also depends on how motivated a listener is to understand what they are hearing (Eckert et al., 2016; Pichora-Fuller et al., 2016; Richter, 2016).

One way the principle of perceptual effort has been assessed experimentally is to test memory for speech that is intelligible, but acoustically degraded. If acoustically-challenging speech is remembered less well, it suggests additional cognitive processing was needed at perceptual stages. Poorer memory for acoustically degraded speech has indeed been shown for single words (Rabbitt, 1968), word pairs (Heinrich et al., 2008), and short stories (Ward et al., 2016). Koeritzer et al. (2018) played participants lists of spoken sentences in multitalker babble. Semantic ambiguity was varied such that half of the sentences contained one or more ambiguous words ("bark" could refer to the sound a dog makes, or to the outer covering on a tree). This semantic manipulation is relevant because sentences with high-ambiguity words require additional processing to interpret (Rodd et al., 2005). After listening to a list of sentences, participants were visually presented with a list of sentences and asked to judge whether a 
sentence had been heard previously, providing an measure of recognition memory (half of the sentences having been previously presented, half not). There were significant effects of noise (with sentences in noise remembered less well) and semantic ambiguity (with high-ambiguity sentences remembered less well), suggesting both acoustic and linguistic challenge rely on domain-general processing resources. Importantly, these factors also interacted with age, with older adults showing increased sensitivity to acoustic clarity, semantic ambiguity, and their interaction.

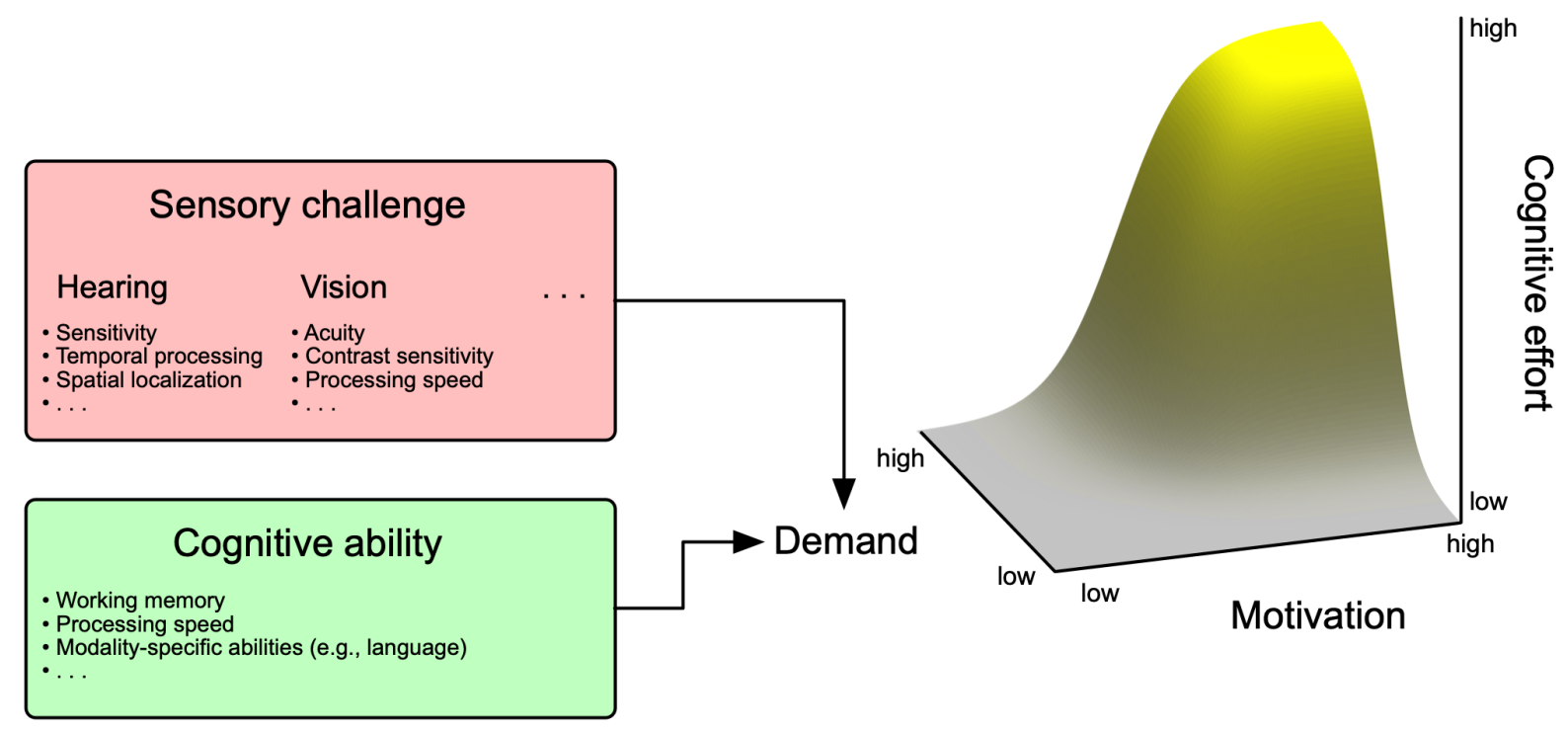

Figure 4.2. Schematic of how sensory challenges and cognitive abilities contribute to cognitive effort. Sensory challenge and cognitive ability jointly determine the perceptual demands in a given situation; when motivation is sufficiently high, this combination can result in increased allocation of cognitive effort (for applications in hearing, see Pichora-Fuller et al., 2016; Peelle, 2018). Available via a CC-BY4.0 license from https://osf.io/rxbtv/.

Additional evidence regarding cognitive consequences of perceptual challenge comes from pupillometry studies. Pupil diameter increases as a function of cognitive load (Kahneman and Beatty, 1966), and thus provides an objective measure of effort while participants complete a task (Laeng et al., 2012; Van Engen and McLaughlin, 2018). In young adults, increased pupil dilation is seen during sentence processing as a function of signal-to-noise ratio (SNR), with larger effects in listeners with poor hearing (Kramer et al., 1997; Zekveld et al., 2010; Zekveld and Kramer, 2014). Although one might expect increased effort in older adults, in some cases older adults' pupil diameters have been shown to be less responsive to changes in SNR than young adults' (Zekveld et al., 2011). It may be that even in quiet listening conditions older adults are already using additional cognitive resources, reducing the effective range of their pupil response.

Converging evidence from a number of sources thus supports the notion that perceptual challenge increases cognitive demand, and can interfere with non-perceptual tasks (such as 
memory). These findings illustrate an important general principle that sensory impairment can potentially have far-reaching effects on other cognitive domains.

\subsubsection{Cognitive Function and Dementia}

One of the more thought-provoking contemporary topics in hearing science relates to whether hearing loss might impact cognitive function. Several epidemiological studies have found that listeners with poorer hearing are at increased risk for cognitive decline and dementia (Lin et al., 2011; Lin et al., 2013). These studies typically look at large samples of older adults who have had both hearing tests (such as pure tone audiometry) and measures of cognition. It is then possible to see whether hearing loss increases the risk for cognitive decline or dementia while statistically controlling for other potential confounds (such as heart disease, hypertension, etc.).

Although the presence of a causal link between hearing loss and cognition is unclear (Wayne and Johnsrude, 2015), one hypothesis is that hearing loss increases reliance on domain general cognitive processes during listening, changing metabolic demands associated with increased pathologic risk. Over time this altered state of network dynamics may contribute to less efficient cognitive processing. A complementary view is inspired by reports that hearing loss is associated with reduced gray matter volume in auditory cortex (Peelle et al., 2011; Eckert et al., 2012), which may also have cascading consequences for network function. Finally, the social effects of hearing loss likely have an important role here: people with hearing loss may be less likely to engage in social situations (such as meeting friends or going out to restaurants) which may have carry-over effects to cognitive function (Chen, 1994; Mick et al., 2014). In the future, results from clinical trials performing randomized interventions (for example, by providing hearing aids to listeners with hearing loss) are one way to better understand the relationship between hearing loss and cognitive decline.

\subsection{Vision}

Although estimates vary, age-related changes in vision are present in $20 \%$ of adults over the age of 65 years (Horowitz, 2004). Age-related changes in visual processing occur at many levels of the visual system, including at the eye (e.g., cataracts or macular degeneration) and visual cortex (Spear, 1993).

\subsubsection{Age-Related Changes in Vision}

\subsubsection{Visual Acuity}

Perhaps the most common association with visual perception is visual acuity: that is, the spatial resolution with which visual stimuli can be resolved. In addition to optical and neural factors of the viewer, visual acuity depends on the spatial contrast (transition between light and dark in an image) and illumination of an image. Although age-related changes in visual acuity occur, declines are modest through age 70 or so, as long as targets are high contrast (that is, a large amount of difference between light and dark in an image, such as black text on a white background) and the conditions are well-lit (Gittings and Fozard, 1986). However, in conditions of low contrast and low luminance, older adults perform more poorly than do young adults (Haegerstrom-Portnoy et al., 1999). Because real-world targets vary in their contrast and illumination, these age-related changes can impact everyday perception. 


\subsubsection{Spatial Contrast Sensitivity}

Spatial contrast is critical for conveying patterns and objects, and sensitivity to this cue can have a significant impact on the perception of targets in everyday vision (Owsley and Sloane, 1987). Typically, spatial contrast threshold is assessed by measuring the amount of contrast a viewer needs to see an object, and can be applied to many different tasks (detection, discrimination, recognition, etc.); contrast sensitivity is the reciprocal of contrast threshold. With aging come a number of physical changes in the optics of the eye that affect contrast sensitivity, including increased light scatter within the eye, that can reduce the perceived contrast of an image (Guirao et al., 1999; Artal et al., 2003). As a result, older adults show increased difficulty processing images with less contrast, particularly at higher spatial frequencies (Derefeldt et al., 1979; Elliott et al., 1989). Owsley and colleagues (1983), for example, tested spatial contrast sensitivity in older adults using static and moving sinusoid gratings (as well as testing for common eye diseases). Participants were instructed to press a button when the pattern became visible on the screen, and release the button when a pattern ceased being visible, with reversals used to estimate contrast at each spatial frequency. There were no differences in sensitivity at low spatial frequencies, but at higher spatial frequencies older adults showed significantly poorer sensitivity than the young adults. In addition, young adults' sensitivity improved more than did the older adults' when the gratings were moving.

One effect of cataracts is greater decreases in spatial contrast sensitivity. Adamsons and colleagues (1992) assessed contrast sensitivity lens clarity in 110 adults, and found lower contrast sensitivity scores at higher frequencies for viewers with increased lens opacity. Supporting the presence of a causal link between lens opacity and contrast sensitivity, patients undergoing cataract surgery show contrast sensitivity scores that return to normal ranges following cataract removal (Rubin et al., 1993).

\subsubsection{Scotopic Function and Dark Adaptation}

Scotopic vision is the type of vision that is supported primarily by rods and occurs under low luminance conditions (in contrast to photopic vision, occurring in well-lit conditions and relying on cones). In everyday situations scotopic vision occurs during situations such as reading menus in dark restaurants or night driving, and is significantly affected in aging. Older adults have repeatedly been shown to have lower sensitivity to light than younger adults, even after they have adapted to darkness. For example, Birren and Shock (1950) measured dark adaptation by testing sensitivity thresholds over a 30 minute period. They found consistently higher thresholds in older adults, as well as a slightly delayed transition time from cone-mediated to rod-mediated vision. Subsequent findings have confirmed both a significant delay in the cone-rod transition, as well as a slowing in rod-mediated dark adaptation (Jackson et al., 1999).

One potential explanation for age-related changes in scotopic function is a decrease in rod photoreceptors, which decline in aging (Curcio et al., 1993). However, the loss of scotopic sensitivity in older adults is observed in areas of the retina where rod loss is minimal, suggesting rod loss alone is probably not responsible for age-related changes in scotopic function (Jackson et al., 1998). Growing evidence instead implicates age-related changes in the visual cycle, the biochemical pathway responsible for rhodopsin regeneration (Lamb and Pugh, 2004). Slowing of the visual cycle is observed in older adults and would lead to slower dark adaptation. 


\subsubsection{Visual Processing Speed}

In everyday situations we often need to make rapid decisions about visual targets, including detection whether an object is present, discriminating between different targets, and so on. Visual processing speed refers to how quickly these judgments can be made.

One approach to studying visual processing speed has been to assess the functional field of view (Sanders, 1970), or useful field of view (UFOV; Ball et al., 1988), as the visual field area in which useful information can be processed without eye or head movements. The useful field of view is an important framework because in time-limited situations, a smaller field of view requires a viewer to perform more fixations in order to scan the same visual scene than a viewer with a larger field of view, and thus slow visual search. Older adults frequently report difficulty with visual search tasks (Kosnik et al., 1988), even when visual acuity is in the normal range.

Ball et al. (1988) used a radial localization task (locating a target away from fixation) in combination with a secondary central task. They found that older adults made more errors on targets appearing at higher eccentricities, consistent with a decline in the useful field of view. (Interestingly, they also found that useful field of view could be increased with a modest amount of practice.) Subsequent studies have shown that performance on useful field of view tests correlate with cognitive ability (Ball et al., 1993; Goode et al., 1998). In addition, the useful field of view is larger in drivers with more experience (Crundall et al., 1999), suggesting it reflects at least some degree of expertise.

\subsubsection{Consequences of Age-Related Changes in Vision}

\subsubsection{Reading}

For many people reading is important for learning, enjoyment, and communication, and can be significantly affected by vision problems associated with normal aging (Ryan et al., 2003). Akutsu and colleagues (1991) tested reading speed in young and older adults. Within character sizes at which participants show the fastest reading, there were minimal age differences in readers with healthy eyes. However, older adults with eye disease (cataracts or retinal detachment) were significantly slowed. In addition, when reading characters outside this range (very small or very large), older adults' reading times were significantly slower, even in the absence of eye disease. Although not directly tested in this study, age-related loss in contrast sensitivity is one explanation for these findings. Lott and colleagues (2001) tested the reading rate of over 500 adults with relatively good high-contrast visual acuity, and found that reading rate decreased with age. However, there was no indication of an age effect once other factors (such as contrast sensitivity, motor ability, and attentional field integrity) were taken into consideration.

Another aspect of reading is the additional cognitive processing that may be required to understand text that is visually degraded. Gao and colleagues (2011) added visual noise to written passages that were read by young adults. They found that the young adults showed reduced conceptual integration at higher levels of visual noise, which they attributed to increased attentional demands of perceptually challenging text. In a subsequent study, the authors found that older adults were more vulnerable to added noise (Gao et al., 2012). Thus, although still a relatively new area of investigation, there may be cognitive effects of visual challenge in reading that parallel those found in speech comprehension (see Figure 4.2). And, just as understanding speech depends on linguistic and cognitive factors beyond the auditory system, reading reflects 
age-related changes in knowledge-based processes and goals that interface with visual ability (Stine-Morrow et al., 2006).

\subsubsection{Driving}

Although driving can help maintain independence for many older adults, automobile accidents pose a serious health risk to drivers and others. Older adults are more likely than young adults to have car crashes (Evans, 1988a; Ball et al., 2006) and when in an accident older adults' injuries tend to be more serious (Evans, 1988b; Barr, 1991). Thus, understanding the role age-related visual changes might play in driving safety is critically important.

Kline and colleagues (1992) surveyed drivers across the lifespan and asked about visual problems when driving, including dealing with unexpected vehicles, vehicle speed, dim displays, windshield problems, and reading road signs. These problems increased with age, with older adults reporting more difficulty along every dimension tested. Although a number of age-related changes might lead to these experiences, linking driving difficulty to specific visual abilities is one way of isolating (and potentially addressing) the underlying mechanisms. In a study focusing on older adult drivers, Ball and colleagues (1993) examined visual processing and driving in adults between the ages of 55 and 90 years. They found that the useful field of view had high sensitivity and specificity in predicting which drivers had experienced car crashes, and was better at predicting crash frequency than other measures (including cognitive status, eye health, and visual sensory function).

Although the useful field of view has received considerable attention, it is also important to note the importance of peripheral vision in driving (in other words, regions outside the "useful field" of view) (Wolfe et al., 2017). For example, input from peripheral vision may help maintain lane position (Summala et al., 1996). Future research will help clarify the relative contributions of central and peripheral vision to driving ability.

In summary, converging evidence indicates that visual changes in aging are associated with increased difficulty with driving and likely contribute to increased crash risks in older adults.

\subsection{Taste, Smell, and Balance}

The focus of this chapter has been on auditory and visual changes, but these are by no means the only modalities that show age-related changes in perception. Smell, taste, and balance are also prone to changing in adult aging and can affect a wide range of everyday activities.

Although relying on different sensory organs, taste and smell are tightly linked and often considered together. Loss of taste and smell are common in older adults (Schiffman, 1997; Attems et al., 2015). The olfactory system undergoes significant changes in aging, including atrophic changes in the olfactory bulb and reductions in the number of receptors in the olfactory epithelium (Hinds and McNelly, 1981). Possible underlying mechanisms include age-related changes in chemosensory signal transduction or gene expression. In addition, several families of medications (that may be consumed more often by older adults) can affect taste and smell (Schiffman, 2018). Changes in smell in particular have been linked to early detection of neurodegenerative conditions such as Alzheimer's Disease (Velayudhan, 2015).

Balance deficits are also common in older age (Agrawal et al., 2009). Although related in part to proprioceptive and visual changes, age-related changes in the vestibular system are also significant contributors (Sturnieks et al., 2008). These include changes to the peripheral vestibular system (otolith organs and semicircular canals) (Johnsson, 1971), and the central 
vestibular system (brainstem and cerebellum) (Lopez et al., 1997; Tang et al., 2001). Problems with gait and balance are associated with increased incidence of falls in older adults (Lipsitz et al., 1991) as well as increased cognitive demand (Brown et al., 1999).

\subsection{Conclusions}

Changes in sensory processing are hallmarks of normal aging and are seen in all modalities. Sensory declines can directly or indirectly affect perception, movement, communication, social engagement, cognitive processing demands, and more. Importantly, significant differences are found across individuals, with some older adults being more impacted by age-related changes to sensory systems than others. Interindividual variability in sensory decline (and the cognitive consequences that follow) is a critical part of understanding adult development and aging.

\section{Acknowledgments}

This work was supported by grant R01DC014281 from the US National Institutes of Health. 


\section{References}

Abel SM, Giguere C, Consoli A, Papsin BC (2000) The effect of aging on horizontal plane sound localization. J Acoust Soc Am 108:743-752.

Adamsons I, Rubin GS, Vitale S, Taylor HR, Stark WJ (1992) The effect of early cataracts on glare and contrast sensitivity. A pilot study. Arch Ophthalmol 110:1081-1086.

Agrawal Y, Carey JP, Della Santina CC, Schubert MC, Minor LB (2009) Disorders of balance and vestibular function in US adults: data from the National Health and Nutrition Examination Survey, 2001-2004. Arch Intern Med 169:938-944.

Akutsu H, Legge GE, Ross JA, Schuebel KJ (1991) Psychophysics of reading--X. Effects of agerelated changes in vision. J Gerontol 46:P325-331.

Anderson S (2017) Clinical Translation: Aging, Hearing Loss, and Amplification. In: The frequency-following response (Kraus N, Anderson S, White-Schwoch T, Fay RR, Popper AN, eds): Springer, Cham.

Anderson S, Parbery-Clark A, White-Schwoch T, Kraus N (2012) Aging affects neural precision of speech encoding. J Neurosci 32:14156-14164.

Anderson S, Parbery-Clark A, White-Schwoch T, Drehobl S, Kraus N (2013) Effects of hearing loss on the subcortical representation of speech cues. J Acoust Soc Am 133:3030-3038.

Artal P, Guirao A, Berrio E, Piers P, Norrby S (2003) Optical aberrations and the aging eye. Int Ophthalmol Clin 43:63-77.

Attems J, Walker L, Jellinger KA (2015) Olfaction and Aging: A Mini-Review. Gerontology 61:485-490.

Ball K, Owsley C, Sloane ME, Roenker DL, Bruni JR (1993) Visual attention problems as a predictor of vehicle crashes in older drivers. Investigative Opthalmology and Visual Science 34:3110-3123.

Ball KK, Beard BL, Roenker DL, Miller RL, Griggs DS (1988) Age and visual search: expanding the useful field of view. J Opt Soc Am A 5:2210-2219.

Ball KK, Roenker DL, Wadley VG, Edwards JD, Roth DL, McGwin G, Jr., Raleigh R, Joyce JJ, Cissell GM, Dube T (2006) Can high-risk older drivers be identified through performance-based measures in a Department of Motor Vehicles setting? J Am Geriatr Soc 54:77-84.

Barr RA (1991) Recent changes in driving among older adults. Hum Factors 33:597-600.

Birren JE, Shock NW (1950) AGe changes in rate and level of visual dark adaptation. J Appl Physiol 2:407-411.

Bolia RS, Nelson WT, Ericson MA, Simpson BD (2000) A speech corpus for multitalker communications research. J Acoust Soc Am 107:1065-1066.

Brown LA, Shumway-Cook A, Woollacott MH (1999) Attentional demands and postural recovery: the effects of aging. J Gerontol A Biol Sci Med Sci 54:M165-171.

Buss E, Hall JW, 3rd, Grose JH (2004) Temporal fine-structure cues to speech and pure tone modulation in observers with sensorineural hearing loss. Ear Hear 25:242-250.

Carlile S, Delaney S, Corderoy A (1999) The localisation of spectrally restricted sounds by human listeners. Hear Res 128:175-189.

Carr CE, Konishi M (1990) A circuit for detection of interaural time differences in the brain stem of the barn owl. J Neurosci 10:3227-3246. 
Chen HL (1994) Hearing in the elderly. Relation of hearing loss, loneliness, and self-esteem. J Gerontol Nurs 20:22-28.

Cruickshanks KJ, Wiley TL, Tweed TS, Klein BE, Klein R, Mares-Perlman JA, Nondahl DM (1998) Prevalence of hearing loss in older adults in Beaver Dam, Wisconsin: The epidemiology of hearing loss study. Am J Epidemiol 148:879-886.

Crundall D, Underwood G, Chapman P (1999) Driving experience and the functional field of view. Perception 28:1075-1087.

Curcio CA, Millican CL, Allen KA, Kalina RE (1993) Aging of the human photoreceptor mosaic: evidence for selective vulnerability of rods in central retina. Invest Ophthalmol Vis Sci 34:3278-3296.

Derefeldt G, Lennerstrand G, Lundh B (1979) Age variations in normal human contrast sensitivity. Acta Ophthalmol (Copenh) 57:679-690.

Dobreva MS, O'Neill WE, Paige GD (2011) Influence of aging on human sound localization. J Neurophysiol 105:2471-2486.

Doty RL (2018) Age-Related Deficits in Taste and Smell. Otolaryngol Clin North Am 51:815825.

Eckert MA, Teubner-Rhodes SE, Vaden KIJ (2016) Is listening in noise worth it? The neurobiology of speech recognition in challenging listening conditions. Ear Hear 37:101S-110S.

Eckert MA, Cute SL, Vaden Jr. KI, Kuchinsky SE, Dubno JR (2012) Auditory cortex signs of age-related hearing loss. JARO 13:703-713.

Elliott DB, Gilchrist J, Whitaker D (1989) Contrast sensitivity and glare sensitivity changes with three types of cataract morphology: are these techniques necessary in a clinical evaluation of cataract? Ophthalmic Physiol Opt 9:25-30.

Evans L (1988a) Older driver involvement in fatal and severe traffic crashes. J Gerontol 43:S186-193.

Evans L (1988b) Risk of fatality from physical trauma versus sex and age. J Trauma 28:368-378.

Fitzgibbons PJ, Gordon-Salant S (2010) Behavioral Studies With Aging Humans: Hearing Sensitivity and Psychoacoustics. In: The aging auditory system (Gordon-Salant S, Frisina RD, Popper AN, Fay RR, eds). New York, NY: Springer.

Gallun FJ, Diedesch AC, Kampel SD, Jakien KM (2013) Independent impacts of age and hearing loss on spatial release in a complex auditory environment. Front Neurosci 7:252.

Gao X, Levinthal BR, Stine-Morrow EAL (2012) The effects of ageing and visual noise on conceptual integration during sentence reading. Quarterly Journal of Experimental Psychology 65:1833-1847.

Gao X, Stine-Morrow EAL, Noh SR, Eskew Jr. RT (2011) Visual noise disrupts conceptual integration in reading. Psychonomic Bulletin and Review 18:83-88.

Gates GA, Mills JH (2005) Presbycusis. Lancet 366:1111-1120.

Gelfand SA, Ross L, Miller S (1988) Sentence reception in noise from one versus two sources: effects of aging and hearing loss. J Acoust Soc Am 83:248-256.

Gittings NS, Fozard JL (1986) Age related changes in visual acuity. Exp Gerontol 21:423-433.

Glyde H, Buchholz JM, Dillon H, Cameron S, Hickson L (2013) The importance of interaural time differences and level differences in spatial release from masking. J Acoust Soc Am 134:EL147-152. 
Goode KT, Ball KK, Sloane M, Roenker DL, Roth DL, Myers RS, Owsley C (1998) Useful field of view and other neurocognitive indicators of crash risk in older adults. J Clin Psychol Med S 5:425-440.

Gordon-Salant S, Yeni-Komshian GH, Fitzgibbons PJ, Barrett J (2006) Age-related differences in identification and discrimination of temporal cues in speech segments. J Acoust Soc Am 119:2455-2466.

Grose JH, Mamo SK (2012) Frequency modulation detection as a measure of temporal processing: age-related monaural and binaural effects. Hear Res 294:49-54.

Guirao A, Gonzalez C, Redondo M, Geraghty E, Norrby S, Artal P (1999) Average optical performance of the human eye as a function of age in a normal population. Invest Ophthalmol Vis Sci 40:203-213.

Haegerstrom-Portnoy G, Schneck ME, Brabyn JA (1999) Seeing into old age: Vision function beyond acuity. Optometry and Vision Science 76:141-158.

Heinrich A, Schneider BA, Craik FIM (2008) Investigating the influence of continuous babble on auditory short-term memory performance. Quarterly Journal of Experimental Psychology 61:735-751.

Hinds JW, McNelly NA (1981) Aging in the rat olfactory system: correlation of changes in the olfactory epithelium and olfactory bulb. J Comp Neurol 203:441-453.

Horowitz A (2004) The prevalence and consequences of vision impairment in later life. Topics in Geriatric Rehab 20:185-195.

Humes LE (1996) Speech understanding in the elderly. Journal of the American Academy of Audiology 7:161-167.

Humes LE, Dubno JR (2010) Factors affecting speech understanding in older adults. In: The aging auditory system (Gordon-Salant S, Frisina RD, Popper AN, Fay RR, eds), pp 211258. New York: Springer.

Jackson GR, Owsley C, McGwin G, Jr. (1999) Aging and dark adaptation. Vision Research 39:3975-3982.

Jackson GR, Owsley C, Cordle EP, Finley CD (1998) Aging and scotopic sensitivity. Vision Res 38:3655-3662.

Johnsson LG (1971) Degenerative changes and anomalies of the vestibular system in man. Laryngoscope 81:1682-1694.

Kahneman D, Beatty J (1966) Pupil diameter and load on memory. Science 154:1583-1585.

Kline DW, Kline TJ, Fozard JL, Kosnik W, Schieber F, Sekuler R (1992) Vision, aging, and driving: the problems of older drivers. J Gerontol 47:P27-34.

Koeritzer MA, Rogers CS, Van Engen KJ, Peelle JE (2018) The impact of age, background noise, semantic ambiguity, and hearing loss on recognition memory for speech. Journal of Speech, Language, and Hearing Research 61:740-751.

Kosnik W, Winslow L, Kline D, Rasinski K, Sekuler R (1988) Visual changes in daily life throughout adulthood. J Gerontol 43:P63-70.

Kramer SE, Ketelyn TS, Festen JM, Kuok DJ (1997) Assessing aspects of auditory handicap by means of pupil dilation. Audiology:155-164.

Kujawa SG, Liberman MC (2015) Synaptopathy in the noise-exposed and aging cochlea: Primary neural degeneration in acquired sensorineural hearing loss. Hearing Research 330:191-199.

Laeng B, Sirois S, Gredebäck G (2012) Pupillometry: A window to the preconscious? Perspect Psychol Sci 7:18-27. 
Lamb TD, Pugh EN, Jr. (2004) Dark adaptation and the retinoid cycle of vision. Prog Retin Eye Res 23:307-380.

Lin FR, Metter J, O'Brien RJ, Resnick SM, Zonderman AB, Ferrucci L (2011) Hearing loss and incident dementia. Archives of Neurology 68:214-220.

Lin FR, Yaffe K, Xia J, Xue Q-L, Harris TB, Purchase-Helzner E, Satterfield S, Ayonayon HN, Ferrucci L, Simonsick EM (2013) Hearing loss and cognitive decline in older adults. JAMA Intern Med 173:293-299.

Lipsitz LA, Jonsson PV, Kelley MM, Koestner JS (1991) Causes and correlates of recurrent falls in ambulatory frail elderly. J Gerontol 46:M114-122.

Lopez I, Honrubia V, Baloh RW (1997) Aging and the human vestibular nucleus. J Vestib Res 7:77-85.

Lott LA, Schneck ME, Haegerstrom-Portnoy G, Brabyn JA, Gildengorin GL, West CG (2001) Reading performance in older adults with good acuity. Optom Vis Sci 78:316-324.

Merchant SN, Nadol JB (2010) Schuknecht's pathology of the inner ear, Third Edition Edition. Shelton, CT: People's Publishing House.

Mick P, Kawachi I, Lin FR (2014) The Association between Hearing Loss and Social Isolation in Older Adults. Otolaryngology-Head and Neck Surgery 150:378-384.

Middlebrooks JC (1992) Narrow-band sound localization related to external ear acoustics. J Acoust Soc Am 92:2607-2624.

Middlebrooks JC, Green DM (1991) Sound localization by human listeners. Annu Rev Psychol 42:135-159.

Moore BC, Peters RW, Glasberg BR (1992) Detection of temporal gaps in sinusoids by elderly subjects with and without hearing loss. J Acoust Soc Am 92:1923-1932.

Owsley C (2016) Vision and aging. Ann Rev Vis Sci 2:255-271.

Owsley C, Sloane ME (1987) Contrast sensitivity, acuity, and the perception of 'real-world' targets. Br J Ophthalmol 71:791-796.

Owsley C, Sekuler R, Siemsen D (1983) Contrast sensitivity throughout adulthood. Vision Res 23:689-699.

Ozmeral EJ, Eddins AC, Frisina DR, Sr., Eddins DA (2016) Large cross-sectional study of presbycusis reveals rapid progressive decline in auditory temporal acuity. Neurobiol Aging 43:72-78.

Peelle JE (2018) Listening effort: How the cognitive consequences of acoustic challenge are reflected in brain and behavior. Ear Hear 39:204-214.

Peelle JE, Wingfield A (2016) The neural consequences of age-related hearing loss. Trends Neurosci 39:486-497.

Peelle JE, Troiani V, Grossman M, Wingfield A (2011) Hearing loss in older adults affects neural systems supporting speech comprehension. J Neurosci 31:12638-12643.

Pichora-Fuller MK, Souza PE (2003) Effects of aging on auditory processing of speech. Int J Audiol 42:11-16.

Pichora-Fuller MK, Schneider BA, Daneman M (1995) How young and old adults listen to and remember speech in noise. J Acoust Soc Am 97:593-608.

Pichora-Fuller MK, Kramer SE, Eckert MA, Edwards B, Hornsby BWY, Humes LE, Lemke U, Lunner T, Matthen M, Mackersie CL, Naylor G, Phillips NA, Richter M, Rudner M, Sommers MS, Tremblay KL, Wingfield A (2016) Hearing impairment and cognitive energy: The framework for understanding effortful listening (FUEL). Ear Hear 37:5S$27 \mathrm{~S}$. 
Rabbitt PMA (1968) Channel capacity, intelligibility and immediate memory. Quarterly Journal of Experimental Psychology 20:241-248.

Richter M (2016) The moderating effect of success importance on the relationship between listening demand and listening effort. Ear Hear 37:111S-117S.

Rodd JM, Davis MH, Johnsrude IS (2005) The neural mechanisms of speech comprehension: fMRI studies of semantic ambiguity. Cereb Cortex 15:1261-1269.

Rosen S (1992) Temporal information in speech: Acoustic, auditory and linguistic aspects. Philos Roy Soc B 336:367-373.

Rubin GS, Adamsons IA, Stark WJ (1993) Comparison of acuity, contrast sensitivity, and disability glare before and after cataract surgery. Arch Ophthalmol 111:56-61.

Ryan EB, Anas AP, Beamer M, Bajorek S (2003) Coping with Age-Related Vision Loss In Everyday Reading Activities. Educational Gerontol 29:37-54.

Sanders AF (1970) Some aspects of the selective process in the functional field of view. Ergonomics 13:101-117.

Schiffman SS (1997) Taste and smell losses in normal aging and disease. Journal of the American Medical Association 278:1357-1362.

Schiffman SS (2018) Influence of medications on taste and smell. World J Otorhinolaryngol Head Neck Surg 4:84-91.

Schneider BA, Pichora-Fuller MK, Kowalchuk D, Lamb M (1994) Gap detection and the precedence effect in young and old adults. J Acoust Soc Am 95:980-991.

Sergeyenko Y, Lall K, Liberman MC, Kujawa SG (2013) Age-related cochlear synaptopathy: An early-onset contributor to auditory functional decline. J Neurosci 33:13686-13694.

Skoe E, Kraus N (2010) Auditory brain stem response to complex sounds: A tutorial. Ear Hear 31:302-324.

Snell KB (1997) Age-related changes in temporal gap detection. J Acoust Soc Am 101:22142220.

Spear PD (1993) Neural bases of visual deficits during aging. Vision Res 33:2589-2609.

Stine-Morrow EA, Miller LM, Hertzog C (2006) Aging and self-regulated language processing. Psychol Bull 132:582-606.

Sturnieks DL, St George R, Lord SR (2008) Balance disorders in the elderly. Neurophysiol Clin 38:467-478.

Summala H, Nieminen T, Punto M (1996) Maintaining lane position with peripheral vision during in-vehicle tasks. Human Factors 38:442-451.

Tang Y, Lopez I, Baloh RW (2001) Age-related change of the neuronal number in the human medial vestibular nucleus: a stereological investigation. J Vestib Res 11:357-363.

Tun PA, Wingfield A (1999) One voice too many: Adult age differences in language processing with different types of distracting sounds. Journals of Gerontology: Series B: Psychological Sciences and Social Sciences 54B:317-327.

Van Engen KJ, McLaughlin DJ (2018) Eyes and ears: Using eye tracking and pupillometry to understand challenges to speech recognition. Hear Res 369:56-66.

Velayudhan L (2015) Smell identification function and Alzheimer's disease: a selective review. Curr Opin Psychiatry 28:173-179.

Walton JP (2010) Timing is everything: Temporal processing deficits in the aged auditory brainstem. Hearing Research 264:63-69. 
Ward CM, Rogers CS, Van Engen KJ, Peelle JE (2016) Effects of age, acoustic challenge, and verbal working memory on recall of narrative speech. Experimental Aging Research 42:126-144.

Wayne RV, Johnsrude IS (2015) A review of causal mechanisms underlying the link between age-related hearing loss and cognitive decline. AGeing Res Rev 23.

Wingfield A, Tun PA, McCoy SL (2005) Hearing loss in older adulthood: What it is and how it interacts with cognitive performance. Current Directions in Psychological Science 14:144-148.

Wolfe B, Dobres J, Rosenholtz R, Reimer B (2017) More than the Useful Field: Considering peripheral vision in driving. Appl Ergon 65:316-325.

Worden FG, Marsh JT (1968) Frequency-following (microphonic-like) neural responses evoked by sound. Electroencephalogr Clin Neurophysiol 25:42-52.

Zekveld AA, Kramer SE (2014) Cognitive processing load across a wide range of listening conditions: Insights from pupillometry. Psychophysiol 51:277-284.

Zekveld AA, Kramer SE, Festen JM (2010) Pupil response as an indication of effortful listening: The influence of sentence intelligibility. Ear Hear 31:480-490.

Zekveld AA, Kramer SE, Festen JM (2011) Cognitive load during speech perception in noise: The influence of age, hearing loss, and cognition on the pupil response. Ear Hear 32:498510. 